\title{
De la propedéutica de los principios cooperativos a la intercooperación como pilastra del cooperativismo
}

\author{
(From the propaedeutics of cooperative principles to \\ intercooperation as a pillar of cooperativism)
}

José Eduardo de Miranda

Director General de Kheíron Educacional y Presidente de la AIDC/BR

Reclbído: 31.05.2014

Aceptado: 30.07.2014

Sumario: I. Introducción. II. La importancia de los Congresos de Viena de 1930 y el de Londres de 1934. III. El Congreso de París de 1937: la enunciación dualista de los Principios Cooperativos. 3.1. Los Principios primarios. 3.2. Los Principios secundarios. IV. El Congreso de Bournemouth de 1963 y la preocupación por impedir la restricción del alcance de los Principios Cooperativos. V. El Congreso de Viena de 1966 y el surgimiento del Principio de la Integración entre Cooperativas. VI. El Congreso de Manchester y el reconocimiento del Principio de la Cooperación entre Cooperativas. VII. A título de conclusión: la Intercooperación es la pilastra del Cooperativismo. VIII. Bibliografía.

Resumen: Este trabajo desarrolla un abordaje sobre el marco de conformación de los Principios Cooperativos, a través del sistema de trabajo que la Alianza Cooperativa Internacional desdobló a lo largo de su historia, en el ámbito de sus principales Congresos. De la misma forma, presenta el momento en el cual el Principio de la Integración entre Cooperativas surge en el seno normativo del Cooperativismo, y cuando pasa a ser reconocido como Principio de la Cooperación entre Cooperativas, o Principio de la Intercooperación.

Palabras clave: Cooperativismo, Principios Cooperativos, Intercooperación.

Abstract: This paper develops an approach on the part of shaping the Cooperative Principles, through the system of work that the International Cooperative Alliance unfolded throughout its history, in the area of the main Congress. Likewise, introduced at which the Principle of Integration between Cooperatives arises in policy within the Cooperative, and when it becomes recognized as a principle of cooperation among cooperatives, or Principle of Intercooperation.

Key words: Cooperative, Cooperative Principles, Intercooperation.

1 Correo electrónico: jemiranda@mirandacorrealima.com 
«Mucha gente entiende los Principios como mandamientos firmes que deben ser seguidos literalmente. En un sentido es verdad, en la medida en que deberían proporcionar normas de conducta. En otro sentido, deberían restringir, incluso prohibir, ciertas acciones mientras estimulan otras»

\section{Alianza Cooperativa Internacional ${ }^{2}$}

\section{Introducción}

Hoy por hoy, nadie duda sobre la importancia de la actuación de los Pioneros de Rochadle en el seno del Cooperativismo mundial. Sin embargo, los Principios que han posibilitado el nacimiento y fortalecimiento de la Sociedad de los Probos se han quedado en el ostracismo hasta muchos años después de su fundación como primera entidad cooperativa «oficial».

Es justo después del nacimiento de la Alianza Cooperativa Internacional-ACl${ }^{3}$ que el mundo del Cooperativismo decide desarrollar un estudio relativo a los auténticos Principios y técnicas de la Cooperación 4 . En el comienzo, la $\mathrm{ACl}$ se propone a fijar un conjunto de Principios con validad universal. Parte de una observación general de las reglas de Rochadle y desencadena unos debates que se prolongan a través de los años ${ }^{5}$, hasta que en el Congreso de Manchester formula el elenco de los Principios Cooperativos que son conocidos por el mundo, hoy.

2 ACl. La Declaración de la Alianza Cooperativa Internacional sobre la Identidad Cooperativa. Anuario de Estudios Cooperativos, Bilbao: Universidad de Deusto, 1995. p. 87.

3 NAMORADO entiende que "o movimento cooperativo percorria o segmento inicial da sua trajetória, no quadro de uma forte interacção com outras componentes do movimento operário, ademais do carácter difuso da explicitação dos princípios e à própria fluidez da atividade cooperativa» (NAMORADO, R. Os Princípios cooperativos. Coimbra: Fora do Texto, 1995, p. 10).

4 GOMEZ enseña que el objetivo de la $\mathrm{ACl}$ es mejorar las condiciones de las clases trabajadoras y proporcionar el establecimiento de relaciones comerciales entre los cooperativistas de los distintos tipos (GOMEZ, L. La alianza cooperativa internacional. Su desarrollo como institución y en especial como instrumento transformador de la sociedad. Vitoria: CSCE-Ekgk, 1998. p. 12).

5 En este sentido, AKE BÖÖK subraya que «a ambição da $\mathrm{ACl}$ em estabelecer uns Principios Cooperativos para o movimento cooperativo mundial tomou como seu ponto de partida as regras de Rochdale. O trabalho começou em 1919, e a questão fo discutida nos Congresos da década de 1920. Em Viena, 1931, um comité especial foi nomeado e as suas recomendações foram finalmente aceites como Princípios em 1937». (AKE BÖÖK, S. Valores cooperativos num mundo de mudanza. Lisboa: Instituto Antonio Sérgio do Sector Cooperativo-Inscoop, 1993. p. 224). 
En este escenario, desarrollase el presente trabajo con el propósito de puntuarse la representatividad de los principales Congresos de la $\mathrm{ACl}$ en el marco de estudios y conformación de los Principios Cooperativos, identificando el momento exacto del surgimiento de la Intercooperación, entonces como Principio de la Integración entre Cooperativas. Asimismo, se va establecer que la Intercooperación, hoy por hoy, revelase como la principal pilastra que sostiene el propio Cooperativismo.

\section{La importancia de los Congresos de Viena de 1930 y el de Londres de 1934}

El Congreso de Viena, realizado por la $\mathrm{ACl}$ en 1930, representa el comienzo de las investigaciones oficiales que buscan una mejor comprensión, o quizá determinación, de los Principios Cooperativos. En esta oportunidad, la Fédération Nationale Française des Cooperatives de Consommation presenta un memorando que al fin del encuentro sirve como base de la resolución que ha decidido que la Alianza debería realizar una investigación entre sus asociados sobre la aplicación corriente de los Principios de Rochdale ${ }^{6}$. Fue entonces cuando se crea la Comisión constituida por Suter, en representación de Suiza; Rapacki, representando a Polonia; Ventosa Roig, por España; E. de Balogh, por Hungría y Warbasse, como representante de Estados Unidos.

El método de trabajo que la Comisión desarrolla consiste básicamente en la redacción de un cuestionario que es contestado por la mayoría de los representantes de organizaciones miembros de la $\mathrm{ACl}$.

De un modo general, dicho cuestionario se ocupa de averiguar sobre seis puntos específicos de los Principios de Rochdale: un hombre, un voto; venta al contado; dividendos sobre las compras; eliminación de beneficios sobre los precios; dividendos limitados sobre el capital, y neutralidad política.

Analizada la encuesta, en el Congreso celebrado en Londres, en el año de 1934, la Comisión propone un informe donde recomienda que la $\mathrm{ACl}$ promueva una modificación en sus estatutos ${ }^{7}$, específicamente

6 WATKINS esclarece que «el Congreso de la ACI pide al Comité Ventral designar una comisión especial para investigar las condiciones en las que son aplicados los Principios de Rochdale en los diversos países y para precisarlos eventualmente» (WATKINS, Pensamiento estático y dinámico en el movimiento cooperativo. Buenos Aires: Intercoop Editora Cooperativa Limitada, 1973, p. 183 - 184).

7 A pesar de que la $\mathrm{ACI}$ no haya publicado este informe, WATKINS afirma que han sido aprobados, y «como resultado de sus recomendaciones, se produjeron ciertas enmiendas en los Estatutos de la Alianza, para asegurar que sólo se admitirían como aso- 
en lo que se refiere al artículo $2 .^{\circ}$, sobre sus propios Principios, y $8 .^{\circ}$, que define las condiciones de afiliación.

Además, ofrece un elenco de siete Principios, cuatro de los cuales identificados como obligatorios y tres, llamados de métodos de acción y de organización. Entre los obligatorios se ha podido encontrar el principio de la adhesión libre; el control democrático, el retorno con base en las compras y el interés limitado al capital. El grupo de los métodos de acción y organización queda constituido por los Principios de la neutralidad política y religiosa, de la venta al contado y del desarrollo de la educación 8 .

El Congreso de 1934 pasa a la historia porque ha sido el primer intento de establecimiento de unos Principios Cooperativos de alcance universal y porque a través de este Congreso se ha comprobado la necesidad de un apoyo doctrinal común en el sistema cooperativo9 .

\section{El Congreso de París de 1937: la enunciación dualista de los Principios Cooperativos}

Concluidos los trabajos realizados en el Congreso anterior, la comisión presenta los resultados de sus investigaciones bajo tres aspectos distintos: primero, el que destaca los Principios de la cooperación tal cual los han practicado los Pioneros de Rochdale; segundo, la actualidad de su aplicación; y tercero, el que se ha ocupado de las conclusiones y recomendaciones ${ }^{10}$. Además, la Comisión añade que las

ciados a la Alianza, aquellas organizaciones que por su constitución y modos de organización, se ajustaron a los Principios de Rochdale» (WATKINS, W. P. Los Principios cooperativos hoy y mañana. Bogotá: Esacoop, 1989. p. 24).

8 DESROCHE anota que además de estos, más cuatro han sido considerados controvertidos: la indivisibilidad o inalienabilidad del activo; transacciones exclusivas con miembros; adhesión voluntaria; venta al precio del mercado (DESROCHE, H. «Des «principes» aux «valeurs». Étapes d'une $\mathrm{ACl}$ revisitée dans son histoire et dans sa préhistoire documentaires» en Communautés - Asscod. 83, París, 1988. p. 27).

9 EGÍA VILLASEÑOR afirma el comité, «aunque no estaba muy convencido de su trabajo, se presentó al Congreso insistiendo de entrada que «no es necesaria ni deseable ninguna modificación de los Principios de Rochdale», y tal vez por eso, como también porque era el primer intento para establecer unos Principios cooperativos de alcance universal, fue que no consiguió la aprobación a su informe por parte de los delegados (EGUIA VILLASEÑOR, F. «Los Principios cooperativos de 1937», EGUIA VILLASEÑOR, F. (Coordinador). Los Principios del cooperativismo. De Rochdale a nuestros días. México D. F.: Confederación Mejicana de Cajas Populares, 1984).

10 En comentario realizado acerca de la repercusión y del sentido del elenco de los siete Principios, DESROCHE esclarece que durante tres docenas de años, el movimiento cooperativo internacional vivirá con base en este elenco más o menos normativo. La nueva nomenclatura va a ser traducida en todas las lenguas y va a ser fijada en las tienISSN: 1134 - 993X, Núm. 48/2014, Bilbao, págs. 149-163 
cooperativas de consumidores se basan, aunque de manera insuficiente e incompleta en los Principios enunciados por los Probos de Rochdale.

Refiriéndose a otras clases de cooperativas, la Comisión insiste que los progresos de la industria y del comercio no han alterado la validez de los siete Principios, pero sí que carecen de una interpretación más rigurosa. Así, la Comisión concluye su tarea fortaleciendo la eficacia de los siete Principios. Sin embargo, afirma que la autenticidad de una cooperativa depende de la adopción y la práctica de los cuatro primeros Principios ${ }^{11}$, es decir: 1. la adhesión libre; 2. el control democrático; 3. la distribución del superávit a los miembros en proporción con sus transacciones, y 4 . el interés limitado sobre el capital» (los tres restantes son: 5 . la neutralidad política y religiosa; 6 . la venta al contado, y 7. la promoción de la educación) ${ }^{12}$.

Los siete Principios han atravesado la Segunda Guerra Mundial, así como la reconstrucción de los países destruidos. Han presenciado el surgimiento de la Organización de las Naciones Unidas y han acompañado la filiación de la $\mathrm{ACl}$ a esta nueva entidad de indiscutible importancia mundial.

De un modo general los Principios Cooperativos aprobados en 1937 han servido de fuente inspiradora para un gran número de cooperativas,

das, en las oficinas, en las aldeas, en las salas de reuniones. Era una relación cómoda y elástica. Simbolizaba una plataforma internacionalista. WATKINS, a su turno, anota que las investigaciones más profundas de la Comisión sobre el funcionamiento de las diversas formas de de la cooperación no desmintieron, sino más bien confirmaron, la tesis de que los siete Principios enumerados en su primer informe no eran solamente específicos de Rochdale o de la simple cooperación de consumo, sino que se aplicaban a todas las formas de cooperación auténtica» (ver sobre el tema en DESROCHE, 1988, p. 30; y WATKINS, 1973, p. 218).

11 Nos parece importante anotar que VALKO ha afirmado que «desde entonces el principio de «puertas abiertas» y los restantes reformulados por la $\mathrm{ACl}$ han sido la "carta magna» oficial para la verdadera cooperación (VALKO, L. "Los Principios rochdaleanos de hoy» en Revista del Instituto de Estudios Cooperativos de la Facultad de Ciencias Económicas de la Universidad nacional de la Plata. 21/22, La Plata, 1963/1964. p. 7-8)

12 CRACOGNA asevera que "el Congreso de París de 1937 efectuó tal declaración de Principios precedida por un relevamiento a escala mundial de las prácticas de las diferentes clases de cooperativas y con un amplio estudio de la comisión especial de eminentes estudiosos designada al efecto. De allí en más quedó claramente establecido, con alcance universal, cuándo una organización podía llamarse genuinamente cooperativa. $\mathrm{Y}$ al mismo tiempo quedó indiscutiblemente sentada la autoridad de la $\mathrm{ACl}$ en materia de doctrina» (CRACOGNA, D. "La Alianza Cooperativa Internacional: pasado, presente y futuro» en Boletín de la Asociación Internacional de Derecho Cooperativo. 28, Bilbao: Universidad de Deusto, 1997. p. 17). 
y sus organizaciones superiores, en el principio de la caminata rumbo al desarrollo de una acentuada participación en el comercio internacional. En el ámbito interno de la $\mathrm{ACl}$ han servido de guía para la creación de departamentos regionales, grupos de trabajo, comités especializados y nuevas estructuras que han formado la base de aproximación entre los cooperadores del Norte y del Sur, del Occidente y del este ${ }^{13}$.

Aunque los siete Principios han sido admitidos, y el informe resulta aprobado casi por mayoría absoluta, algunos doctrinarios han subdividido el elenco en primarios y secundarios y otros entendieron que la lista debía ampliarse, presentando los Principios de diferente manera y a través de un orden distinto.

\section{III.1. Los Principios primarios}

Los Principios primarios han sido elegidos por la $\mathrm{ACl}$ como aquellos que van a identificar la entidad cooperativa y servir de prerrequisito para la afiliación de los nuevos miembros.

Los Principios primarios han sido denominados ${ }^{14}$ : Libre adhesión (puerta abierta); Control democrático (un hombre, un voto); Distribución a los asociados del excedente a prorrata de sus operaciones; Interés limitado sobre el capital; Pago de interés limitado al capital de los socios.

\section{III.2. Los Principios secundarios}

Para identificar a estos Principios la $\mathrm{ACl}$ aprueba una resolución que dice que «aun cuando forman parte, sin la menor duda, del sistema rochdaleano y han sido aplicados exitosamente por los movimientos cooperativos de diversos países, no constituyen, sin embargo, una condición de adhesión de la $\mathrm{ACl}\rangle^{15}$.

El Comité Central de la Alianza ha considerado los Principios secundarios más «como métodos esenciales de acción y organización que como reglas cuyo no cumplimiento destruiría el carácter cooperativo de

13 SALAZAR LEITE, J. Enquadramento histórico-social do movimento cooperativo. Lisboa: Instituo António Sérgio, 1994, p. 181.

14 Sin hacer cualquier diferencia entre primarios y secundarios, SOLDEVILLA Y VILLAR anota que los cuatro primeros Principios han sido aprobados casi por unanimidad de todos los congresistas internacionales. Ya ARANZADI adopta la clasificación y se los presenta a los Principios Cooperativos de 1937 en primarios y secundarios. (Conforme SOLDEVILLA Y VILLAR, 1973, p. 175; y ARANZADI, D.: 1976, p. 75).

15 Alianza Cooperativa Internacional. Compte Rendí du Quinzième Congrès de l'Alliance Coopérative Internationale à Paris, du 6 au 9 septembre 1937. Londres: $\mathrm{ACl}$, 1938. 
una sociedad» ${ }^{16}$. Esos han sido definidos como: Neutralidad política y religiosa; La venta al contado y Desarrollo de la educación.

\section{El Congreso de Bournemouth de 1963 y la preocupación por impedir la restricción del alcance de los Principios Cooperativos}

Pocos años después del Congreso de París, de 1937, explota la Segunda Guerra Mundial, y con su fin la humanidad se ve hundida bajo el doloroso y difícil proceso de recuperación que provoca una profunda modificación geopolítica de la escena mundial ${ }^{17}$.

Durante los años de 1950 las cooperativas han experimentado una metamorfosis de adaptación en el marco de una economía dinámica. En esta época, mientras el movimiento cooperativo ha iniciado una nueva era, fresca y vigorosa ${ }^{18}$. Dadas las características que hemos acabado de narrar, cuando la $\mathrm{ACl}$ llega al Congreso de Bournemouth, en 1963, el cuarto de siglo que ha pasado desde el Congreso de París ha generado un mundo distinto, cuya realidad se encarga por si sola de presionar la eficacia y el sentido de los Principios Cooperativos.

Klimov, cooperativista ruso que representa a Centrosoyuz, pide una resolución que reexamine a los Principios Cooperativos, pues entiende que ya no son universales y solamente representan una adecuación del cooperativismo al capitalismo. Su petición es aceptada y la $\mathrm{ACl}$ designa una Comisión que se encarga de elaborar los Principios de base de la actividad de la cooperación, según el contexto situacional del momento

16 Sobre esta consideración, WATKINS explica que «la distinción expuesta por el Comité entre «métodos» y «normas», parece válida en un sentido, pero no en otro. Mal podría negarse que los primeros cuatro Principios son absolutamente vitales para el carácter cooperativo de cualquier sociedad o federación. A pesar de todo, son tan «métodos» como los otros tres. En realidad los siete estarán mejor denominados como Reglas prácticas que, si se cumplen eficiente y fielmente, aseguran que la sociedad preserve su carácter cooperativo -y si no constituye un triunfo comercial, al menos evitará algunas de las causas más comunes de fracaso» (WATKINS, 1989, p. 25-26).

17 Después del Congreso de París el mundo se ha visto envuelto en trastornos que han abalado casi toda la población del Planeta. "Poucos anos depois, eclodiu a 2. ${ }^{\circ}$ Guerra Mundial, a que se seguiu uma dolorosa recuperação e donde resultou uma profunda alteração geopolítica da cena mundial. A guerra fria agudizou-se no decurso dos anos 50. No quadro de um impetuoso processo de descolonização, novos países surgiram no concerto das nações» (NAMORADO, 1995, p. 13).

18 VALKO, 1963, p. 8. 
y del futuro que se presentaba ${ }^{19}$. Constituida por Bonner, en representación de Gran Bretaña; Karve, venido de India, que representaba a los países en desarrollo; Cowden, norteamericano que respondía por todo el Hemisferio occidental; Henzler, de Alemania, que representaba a Europa Occidental, y Blank, de Rusia, la comisión asume el encargo de contestar a las siguientes tres interrogantes de suprema importancia para el futuro del Cooperativismo:

- ¿Cuáles son los Principios de los Pioneros de Rochdale que han conservado la actualidad y la importancia para la época en que se encontraba el mundo?

- ¿Cuáles deben ser modificados y de qué manera, para contribuir lo más posible a la realización de las tareas que le corresponden al movimiento cooperativo?

- ¿Cuáles de estos Principios han perdido importancia y deben ser substituidos por otros?

El plan de trabajo se ha desarrollado a partir de la observación sobre la aplicación actual de los Principios de Rochdale tal y como han resultado del Congreso de la $\mathrm{ACl}$, de 1937. Se ha formulado un cuestionario que ha sido remitido a las organizaciones filiadas a la $\mathrm{ACl}$, entidades no filiadas y también a personas particulares seleccionadas de acuerdo con su participación, actividad y conocimiento acerca del Movimiento Cooperativo. Esta información nos parece importante puesto que, para responder a las preguntas que le han sido encargadas, la Comisión se ha esforzado por tomar en cuenta los puntos de vista de los cooperadores prácticos, acentuando en numerosos casos el espíritu antes que la letra de cualquier principio. De este modo, ha elaborado una definición práctica que revela los Principios Cooperativos como actos indispensables al logro de los fines del propio movimiento cooperativo.

Asimismo, bajo la fuerza de los objetivos que los Pioneros de Rochdale han establecido en sus estatutos, la Comisión entiende que «en todos los tiempos el elemento común ha sido el hecho de que los mejores propósitos de la cooperación van más allá de promover simplemente los intereses individuales de sus socios. Su finalidad es más bien promover el progreso y el bienestar de la humanidad. Es este objetivo

19 «O Congresso da $\mathrm{ACl}$ de 1963 decidiu encarregar o Comité Central de nomear uma Comissão, cujo mandato seria o de estudar a presente aplicação dos princípios cooperativos nos diferentes tipos de cooperativas e sob os diferentes sistemas económicos e políticos, emitindo recomendações sobre a judiciosa definição que conviria das aos princípios cooperativos em função de sua aplicação na hora actual no mundo inteiro» (SALAZAR LEITE, J. Princípios Cooperativos: a formulação de Viena. Lisboa: Instituto Antonio Sérgio do Sector Cooperativo, 1987. p. 1-2). ISSN: 1134 - 993X, Núm. 48/2014, Bilbao, págs. 149-163 
el que diferencia una cooperativa de una empresa común, y que justifica que sea puesta a prueba no solamente desde el punto de vista de su eficiencia comercial, sino también de su contribución a los valores sociales y morales que elevan la vida humana sobre aquello que es puramente material y animal» 20 .

Respeto a los Principios, los resultados han definido que es posible establecer grados de validez entre los Principios Cooperativos esenciales. Al contrario de priorizar a determinados Principios, la Comisión esclarece que cada uno de ellos constituye un elemento fundamental, igualmente importante, y que necesita ser observado en la medida y como las circunstancias lo permitan, en todo momento y en todos los lugares.

\section{El Congreso de Viena de 1966 y el surgimiento del Principio de la Integración entre Cooperativas}

Las conclusiones de la Comisión constituida en el Congreso de París han sido desarrolladas con el objeto de identificar unos valores esenciales de la Cooperación, que sirvan de base orientadora para los cooperadores que tienen que luchar contra las dificultades para poder aprovechar las ventajas que el mundo moderno ofrece.

Consecuente con todo ello, la Comisión presenta un informe al Comité Central de la Alianza, recomendando que su trabajo sea aprobado sin modificaciones ${ }^{21}$. La aceptación del informe de la Comisión corresponde a la afirmativa de la $\mathrm{ACl}$ en el sentido de que la «reformulación de los Principios Cooperativos mantiene las ideas fundamentales de los Pioneros, agregando algunos matices nuevos que surgen de la experiencia viva y de las cambiantes condiciones de la economía mundial» 22 . Es en sentido de proporcionar una nueva posibilidad de las Cooperativas enfrentar en conjunto las tendencias y dificultades provocadas por los cambios del mundo, el Congreso de Viena de 1966, incorpora la Integración o Cooperación entre Cooperativas, como uno de los Principios Cooperativos que se muestran presentes en el estatuto de la $\mathrm{ACl}$.

20 SALAZAR LEITE, 1987, p. 15.

21 No obstante la aprobación del informe, el Congreso decide enmendar el texto, incluyendo la palabra «racial», en la lista de las discriminaciones indeseables (Véase WATKINS, 1973, p. $448-450$ ).

22 SAN PEDRO, J. Descubramos el cooperativismo en nosotros. Buenos Aires: Intercoop Editora Cooperativa Limitada, 1977. p. 37. 
La formulación de este Principio está conectada a la importancia que los Pioneros de Rochdale han atribuido a la estrecha colaboración entre entidades de fines semejantes que planean la transformación económico-social. Esta idea está registrada en la segunda parte del párrafo 6. ${ }^{\circ}$, del artículo y del primer estatuto., y deja evidente que los Pioneros han planeado el establecimiento de una colonia que se baste por sí misma y en la que se unirán los intereses para la ayuda a otras sociedades ${ }^{23}$.

Este espíritu se confirma con la creación, en 1850, de la Sociedad del Molino Harinero, creada en el propio pueblo de Rochdale, con la colaboración de hombres y dinero venidos de la Cooperativa de los Probos Pioneros.

De la misma forma, en 1863, han impulsado la fundación de la Cooperative Wholegran Society, conocida como la Sociedad Cooperativa del Norte de Inglaterra para la Venta al por Mayor ${ }^{24}$. La constitución de esta ultima entidad es de gran interés, desde el punto de vista cooperativo, porque sirve para unir a todas las cooperativas detallistas, o sea a la distribución o comercio, y luego organizan o controlan otras fábricas e industrias o auxiliares emprendiendo así la cooperatización de la industria.

Cuando han anotado en el párrafo $6 .^{\circ}$ del artículo primero que estaban dispuestos a prestar ayuda para otras sociedades, los Probos de Rochdale han querido decir que se deben unir en un esfuerzo común para realizar los objetivos y los ideales de la totalidad de la Cooperación en sí misma25.

\section{El Congreso de Manchester y el reconocimiento del Principio de la Cooperación entre Cooperativas}

Durante el último tercio del siglo $\mathrm{xx}$, las entidades cooperativas han experimentado una serie de dificultades que han culminado con

23 El párrafo sexto del primer artículo del estatuto de la Sociedad de los Probos de Rochdale establece que «Desde el omento que sea posible esta sociedad emprenderá la organización de las fuerzas de la producción, del a distribución, del a educación y del gobierno, o, dicho en otras palabras, es establecimiento de una colonia que se baste a sí misma y en la que se unirán los intereses, o bien prestará ayuda a otras sociedades para establecer colonias de esa clase». (LAMBERT, P. La doctrina cooperativa. 2. ${ }^{a}$ ed., Buenos Aires: Intercoop Editora Cooperativa Limitada, 1965, p. 327).

24 Véase CORVALAN, 1985, p. 95 y 96; JACOBUS HOLYOAKE, 1944, p. 103; RIVERA CAMPOS, J. El secreto de Rochadle. Buenos Aires: Intercoop Editora Cooperativa Limitada, 1973. p. 46-49; y MLADENATZ, 1969, p. 84 y 85.

25 ALIANZA COOPERATIVA INTERNACIONAL. Nuevos enfoques de los Principios cooperativos en el mundo. Rosario: Ediciones Idelcoop, 1981. p. 55. 
el freno de su proceso de desarrollo. La necesidad de adaptación a un nuevo escenario económico mundial y a unos mercados más competitivos, y la existencia de una serie de retos globales con que la Humanidad debe afrontar en el futuro inmediato ${ }^{26}$, han obligado a replantear la validez de los Principios Cooperativos vigentes, así como el perfil que la sociedad cooperativa debe asumir en el momento actual.

Consciente de la frágil realidad por la cual atraviesa el movimiento cooperativo, y ante la responsabilidad que tiene como la institución de más fuerza del Cooperativismo, la $\mathrm{ACl}$ aprovecha el Congreso de Estocolmo, realizado en el año de 1988, para exponer las lagunas de los Principios Cooperativos vigentes y adoptar una nueva formulación.

Junto a lo anterior la $\mathrm{ACl}$ formula una definición de cooperativas y sanciona el dictamen relativo a la Declaración sobre la Identidad Cooperativa. Además, edita los Valores Cooperativos y reformula los Principios Cooperativos $^{27}$, poniendo de manifiesto que ellos constituyen las «pautas mediante las cuales las cooperativas ponen en práctica sus valores» 28 .

Definidos los Principios, la $\mathrm{ACl}$ se ha ocupado de reformular aquellos que han sido aprobados en el Congreso de Viena, celebrado en el año de 1966. Como fundamento de esta enunciación, explica que entre 1970 y 1995 la economía de mercado ha extendido mucho su impacto por todo el mundo. Además, las barreras comerciales tradicionales habían sido drásticamente oprimidas y muchos cambios, tales como la creación de zonas de libre comercio, la reducción en el apoyo gubernamental para la agricultura, la desregulación de las entidades financieras, amenazaban los marcos económicos dentro de los cuales muchas cooperativas habían funcionado durante décadas.

Para progresar, en muchos casos, simplemente para subsistir, las cooperativas han tenido que examinar cómo iban a reaccionar frente a estas circunstancias modificadas ${ }^{29}$. El resultado del examen ha revelado que «los Principios, que constituyen el centro de las cooperativas, no son independientes el uno del otro. Están unidos sutilmente; cuando se pasa uno por alto, todos se resisten. No hay que evaluar las cooperativas ex-

26 JULIÁ IGUAL, J. F. y GALLEGO SEVILLA, L. P. «Principios cooperativos y legislación de la sociedad cooperativa española. El camino hacia el fortalecimiento de su carácter empresarial» en Revista de Estudios Cooperativos. 70, 2000. p. 131-132.

27 «La quintaesencia del ideario cooperativo desde los Estatutos de la Cooperativa de Rochdale» (VICENT CHULIÁ, F. Compendio crítico de derecho mercantil. 2. ${ }^{a}$ ed. T 1. Barcelona: Librería Bosch, 1986. p. 563. 564).

$28 \mathrm{ACl}$. Declaración de la Alianza Cooperativa Internacional sobre la Identidad Cooperativa aprobada en Manchester. Vitoria-Gasteiz: Consejo Superior de Cooperativas de Euskadi, 1996, p. 17.

29 ALIANZA COOPERATIVA INTERNACIONAL, 1996, p. 27. 
clusivamente en base a un principio dado; más bien, deben ser evaluadas en función de cómo se adhieren a los Principios en su totalidad» ${ }^{30}$.

Dada esta realidad, la Alianza ha establecido que los Principios pierden la singularidad reglamentaria, y ganan un perfil paradigmático, puesto que a la vez representan un modelo de pautas para evaluar el comportamiento y para tomar decisiones. De esta forma, el Congreso de Manchester concluye, pues, que los Principios Cooperativos son «pautas mediante las cuales las cooperativas ponen en práctica sus valores» ${ }^{31}$.

Asimismo, merece ser resaltado que Watkins considera a los Principios Cooperativos como «las ideas generales que inspiran y gobiernan la aplicación de la técnica cooperativa de organización social. Estas ideas resultan del razonamiento inductivo sobre la experiencia de realidades sociales universales». Establece, pues, que los Principios Cooperativos son «líneas para la solución cooperativa de los problemas sociales a los cuales estas realidades dan nacimiento ${ }^{32}$. En el vasto mundo del Cooperativismo, los Principios son comunes a los diversos tipos de clases de cooperativas y formas de cooperación en todo tiempo y lugar.

Bajo esta concepción el Congreso de Manchester reconoce el Principio de la Cooperación entre Cooperativas, y la Alianza Cooperativa Internacional determina que «las cooperativas sirven a sus socios lo más eficazmente posible y fortalecen el movimiento cooperativo trabajando conjuntamente mediante estructuras locales, nacionales, regionales e internacionales» ${ }^{33}$.

El principio está enunciado de manera genérica y nos deja creer que dicha cooperación puede ser generada promoviéndose o no un vínculo patrimonial entre las entidades que participan del proceso colaborador.

Es también conocido como principio de intercooperación, y tiene la finalidad de promover la colaboración entre las sociedades cooperativas, para intentar mejorar su desarrollo y los intereses de sus socios, mediante la solidificación de estructuras conjuntas en todos los ámbitos: regional, nacional e internacional. «De hecho, las cooperativas solamente pueden propagar su impacto mediante la colaboración práctica y rigurosa la una con la otra. Además de poder conseguir reconocido logros a nivel local, deben luchar continuamente para obtener los beneficios de organizaciones a gran escala a la vez que mantienen las ventajas de implicación y asentamiento locales» ${ }^{34}$.

\footnotetext{
30 Ibíd, p. 45.

31 Ibíd, p. 17.

32 WATKINS, 1989, p. 31-32.

33 ALIANZA COOPERATIVA INTERNACIONAL, 1996, p. 63.

34 ALIANZA COOPERATIVA INTERNACIONAL, 1995, p. 96.
} 
La ACl afirma que la colaboración entre cooperativas es un reto perpetuo para todas las entidades del sector cooperativo. Por esto, «las cooperativas deben reconocer, incluso más que en el pasado, la necesidad de reforzar sus organizaciones y actividades de apoyo ${ }^{35}$.

\section{A título de conclusión: la Intercooperación es la pilastra del Cooperativismo}

De acuerdo como la propia Alianza Cooperativa Internacional instituyó por la Declaración sobre la Identidad Cooperativa, en Manchester, en el año de 1995, «no siempre es fácil ver que hay un interés cooperativo general, basado en el valor de la solidaridad y el principio de la cooperación entre cooperativas» ${ }^{36}$.

No siempre es fácil para los actores del Cooperativismo comprender que su propia supervivencia está condicionada al reconocimiento y aplicación de sus valores y principios. Es, pues, imprescindible reconocerse que la intercooperación, o cooperación entre cooperativas, es el paso natural e indispensable para el alcance d los objetivos que son propios de cada Sociedad Cooperativa.

La Intercooperación es el desarrollo básico del Cooperativismo, por cuanto una Sociedad Cooperativa se desarrolla para servir a sus miembros, la cooperación entre Cooperativas es el medio por lo cual suman sus acciones y unifican sus esfuerzos para trabajar conjuntamente y celebrar sinergias con objetivos comunes.

Con este Principio "se pretende, por una parte, que el Cooperativismo no sea hecho aislado relativo a cada una de las cooperativas, sino que el ser cooperativo sea un fenómeno acabado en la medida en que, transcendiendo el ámbito de la cooperativa específica, se prolonga en todas las demás. Pero, también, a través de este principio, se persiguen objetivos económicos, más allá de los estrictamente políticos o representativos, para a través de esa cooperación entre cooperativas conseguir una mayor dimensión empresarial y una mayor competitividad, ante el fenómeno de la dimensión de la empresa creciente y del tamaño mayor de los mercados y la internacionalización de la economía» ${ }^{37}$.

35 Ibíd, p. 97.

36 ALIANZA COOPERATIVA INTERNACIONAL, 1995, p. 96.

37 MARTÍNEZ CHARTERINA. La cooperativa como empresa y los principios cooperativos tras el congreso centenario de la A C I de Manchester in Evolución del escenario económico. Transformaciones en el actual escenario económico y sus proyecciones de futuro. Bilbao: Universidad de Deusto, 1996. p. 223. 
Siguiendo esta idea, podemos decir que la cooperación entre cooperativas ha sido pensada para aproximar las Cooperativas, de modo que trabajen en conjunto, como manera de garantizar que las entidades logren el objetivo de satisfacer las necesidades de sus miembros.

Esta meta ha sido observada por los Probos de Rochdale, cuando, a través del párrafo sexto del artículo primero del estatuto de 1845, determinan que la sociedad prestará ayuda a otras sociedades para establecer colonias de clase idéntica a la suya. El logro de este objetivo, hemos visto, ha sido alcanzado a través del auxilio humano y económico, que han destinado a la constitución de entidades cooperativas tanto en Rochdale, como en otras regiones.

La idea de colaboración entre Cooperativas, que los Probos Pioneros de Rochdale registraron en sus estatutos es, quizás, la fórmula adecuada al fortalecimiento del propio Cooperativismo, lo que se consigue alcanzar a través de la conformación de un ejercicio conjugado y de la formación de redes de actuación entre Sociedades Cooperativas, que pueden, a su tiempo, intercambiar tecnologías, modelos de negocios, paradigmas gerenciales y técnicas de formación cooperativa que valorice el espíritu de la cooperación.

Más allá de una perspectiva utópica, los Cooperativistas deben comprender que la cooperación entre personas se completa con la Intercooperación, con la cooperación entre Sociedades Cooperativas ${ }^{38}$.

Esta es la lógica del Cooperativismo: su pilastra, el eje de supervivencia.

\section{Bibliografía}

AKE BÖÖK, S. Valores cooperativos num mundo de mudanza. Lisboa: Instituto Antonio Sérgio do Sector Cooperativo-Inscoop, 1993.

ALIANZA COOPERATIVA INTERNACIONAL. Nuevos enfoques de los Principios cooperativos en el mundo. Rosario: Ediciones Idelcoop, 1981.

- La Declaración de la Alianza Cooperativa Internacional sobre la Identidad Cooperativa. Anuario de Estudios Cooperativos, Bilbao: Universidad de Deusto, 1995.

ARANZADI TELLERÍA, D. Cooperativismo industrial como sistema, empresa y experiencia. Bilbao: Universidad de Deusto, 1976.

CRACOGNA, D. «La Alianza Cooperativa Internacional: pasado, presente y futuro» en Boletín de la Asociación Internacional de Derecho Cooperativo. 28, Bilbao: Universidad de Deusto, 1997.

38 ARANZADI TELLERÍA, D. Cooperativismo industrial como sistema, empresa y experiencia. Bilbao: Universidad de Deusto, 1976. P. 93. 
DESROCHE, H. «Des «principes» aux «valeurs». Étapes d'une ACI revisitée dans son histoire et dans sa préhistoire documentaires» en Communautés-Asscod. 83, París, 1988.

EGUIA VILLASEÑOR, F. «Los principios cooperativos de 1937», EGUIA VILLASEÑOR, F. (Coordinador). Los principios del cooperativismo. De Rochdale a nuestros días. México D. F.: Confederación Mejicana de Cajas Populares, 1984.

GOMEZ, L. La alianza cooperativa internacional. Su desarrollo como institución y en especial como instrumento transformador de la sociedad. Vitoria: CSCE-Ekgk, 1998.

JULIÁ IGUAL, J. F. y GALLEGO SEVILLA, L. P. «Principios cooperativos y legislación de la sociedad cooperativa española. El camino hacia el fortalecimiento de su carácter empresarial» en Revista de Estudios Cooperativos. 70, 2000.

LAMBERT, P. La doctrina cooperativa. 2. ${ }^{a}$ ed., Buenos Aires: Intercoop Editora Cooperativa Limitada, 1965.

MARTÍNEZ CHARTERINA. La cooperativa como empresa y los principios cooperativos tras el congreso centenario de la A C I de Manchester in Evolución del escenario económico. Transformaciones en el actual escenario económico y sus proyecciones de futuro. Bilbao: Universidad de Deusto, 1996.

NAMORADO, R. Os principios cooperativos. Coimbra: Fora do Texto, 1995.

SALAZAR LEITE, J. Enquadramento histórico-social do movimento cooperativo. Lisboa: Instituo António Sérgio, 1994.

- Princípios Cooperativos: a formulação de Viena. Lisboa: Instituto Antonio Sérgio do Sector Cooperativo, 1987.

SAN PEDRO, J. Descubramos el cooperativismo en nosotros. Buenos Aires: Intercoop Editora Cooperativa Limitada, 1977.

VALKO, L. "Los principios rochdaleanos de hoy» en Revista del Instituto de Estudios Cooperativos de la Facultad de Ciencias Económicas de la Universidad nacional de la Plata. 21/22, La Plata, 1963/1964.

VICENT CHULIÁ, F. Compendio crítico de derecho mercantil. 2. a ed. T 1. Barcelona: Librería Bosch, 1986.

WATKINS, Pensamiento estático y dinámico en el movimiento cooperativo. Buenos Aires: Intercoop Editora Cooperativa Limitada, 1973.

- Los principios cooperativos hoy y mañana. Bogotá: Esacoop, 1989. 